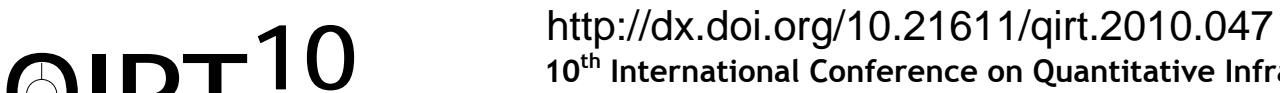 \\ $10^{\text {th }}$ International Conference on Quantitative InfraRed Thermography \\ July 27-30, 2010, Québec (Canada)
}

\section{Infrared Face Recognition: A Review of the State of the Art}

\author{
by Reza Shoja Ghiass, Abdelhakim Bendada and Xavier Maldague
}

\begin{abstract}
Computer Vision and Systems Laboratory, Laval University, Quebec City (Quebec) G1V 0A6, Canada, reza.shoja-ghiass.1@ulaval.ca,bendada@gel.ulaval.ca,maldagx@gel.ulaval.ca
\end{abstract}

\begin{abstract}
In this paper a review of the state of the art has been presented on Infrared face recognition. A comprehensive review of the state of the art has been already done in [1] in 2008. Our review is a complement of the mentioned work [1] with more emphasis given to more recent or more important publications. Initially, we will review the basic important works on Infrared Face Recognition before 2008. Afterwards, we will focus on the recent works which are not reviewed in [1]. Finally, we will draw the conclusions.
\end{abstract}

\section{Introduction}

Face recognition has become one of the most attractive research areas among the researchers from research fields such as image processing, pattern recognition, neural networks, computer vision, computer graphics, and psychology because of the wide range of commercial and law enforcement applications and the availability of feasible technologies after 30 years of research in visible face recognition [2]. As we know, very reliable biometrics such as fingerprint, retina and iris exist. The question, therefore, is which advantages make Face biometric to be superior compared to the other ones? The answer is that Face recognition is unobtrusive. So, it doesn't rely on the cooperation of the subject so, the system can recognize the identity of the person without his/her knowledge. As a result, the overall identification procedure can be also decreased.

Visible face recognition suffers from some severe problems such as the illumination dependency problem which significantly decrease the efficiency of the system, especially, in the outdoor applications. To overcome this limitation, several solutions have been investigated. One solution is using 3D data obtained from 3D vision devises. Such systems are less dependent to the illumination changes. They can also work with rotated faces in multi-view applications. But they have some disadvantages: The cost of such systems is high, their processing speed is low and some artifacts are produced due to speculation [3]. By the way, they are not able to easily handle the facial expression changes.

Another solution for the mentioned limitation is using infrared facial images. The infrared spectrum is divided into four bandwidths: Near-IR (NIR), Short-wave-IR (SWIR), Medium-wave-IR (MWIR) and Long-wave IR (Thermal IR). Out of the four bandwidths Thermal IR has received the most attention in the literature. In the past years, NIR has also received some attention, but SWIR and MWIR have not been investigated very well. Thermal IR sensors measure the emitted heat energy from the object and they don't measure the reflected energy. Thermal spectrum has some advantages: Thermal images of the faces can be obtained under every light condition even under completely dark environments. By the way, they are somewhat robust to facial expression. Thermal energy emitted from the face is less affected to the scattering and the absorption by smoke or dust than reflected visible light. Thermal IR images also reveal anatomical information of face that makes it capable to detect disguises [4]. But, Thermal face recognition suffers from some problems. It is subject to environmental temperature, emotional, physical and health conditions. Drinking alcohol can also affect the thermal image of face. Another problem of the thermal spectrum is the opaqueness to eyeglasses. This makes a large portion of the face wearing eyeglasses to be occluded in thermal images. So, some information around the eyes will be loosed. Each of the mentioned problems has opened a new research line on the thermal face recognition to overcome the thermal face recognition limitations which even now are the lines of research: some researchers have suggested fusing the information from thermal and visual to improve the face recognition rates and solve the opaqueness problem [5]. There are two general categories for Fusion: Decision fusion and data fusion. In the decision fusion, scores obtained from each modality are combined to obtain the total score, while in the data fusion the fusion is done by using a combination of pixel values in the facial images obtained from the two modalities. A simple example for data fusion is a weighted sum of pixel intensity values obtained from each sensor data. Some other researchers have proposed to get the thermal face images and extract the vascular networks [6], [7] while the others have proposed the use of blood perfusion data [8] in order to overcome the ambient temperature dependency problem in thermal face recognition. 
Because of the mentioned problems with thermal face recognition, and the illumination dependency problem of the visible face recognition, some researchers have proposed using active near infrared in face recognition. The Near-IR band is the reflective part of the infrared spectrum. It falls between the visible light band and the thermal infrared band. So, it has the advantages of both visible light and thermal infrared. An important property of NIR spectrum is its invisibility to human eyes. This property makes active Near-IR illumination unobtrusive [9]. An important research field in NIR face recognition has been introduced by Pan et al. [10] by using hyper spectral images in NIR band. Recently the researchers have developed the use of multi spectral images to visible face recognition to cope the illumination dependency problem of visible face recognition [11].

In this paper we review all of the mentioned aspects with more emphasis given to more recent or more important publications. The first review on infrared face recognition has been done by Kong et al. [5]. A more recent review of the state of the art on IR Face Recognition has been done by Akhloofi and Bendada [1]. In our study a complement review of the state of the art for [1] has been done on Infrared face recognition. The outline of this paper is as follows: in section 2 we review the initial important works on Infrared face recognition done before 2008. In section 3 we study some of the important works which have been published after 2008 and are not reviewed in [1]. Finally, we will draw the conclusions in section 4.

\section{A brief review of literature before 2008}

In this section we give a brief review to the important publications in the literature before 2008. We review this section in two parts. Firstly, we review the early publications before 2000. Secondly, we study the important works on infrared face recognition published after 2000.

\subsection{The early publications on Infrared Face recognition (1992-2000)}

The first work on thermal face recognition was conducted in MIKOS Corporation [12] in 1992. MIKOS Corporation demonstrated that the facial thermograms are unique for each individual. In this context, Prokoski et al. patented the use of thermal images for face recognition by analyzing the elemental Shapes in facial thermograms [13]. Wilder et al. [14] presented one of the first works on infrared face recognition. They compared the relative performances of three face recognition algorithms for visible and IR images. They also computed the performance of their system when the visible and infrared information are fused. In their work, they did not consider significant illumination changes for visible images, and temperature changes for IR images. Their work was the first work on fusion of infrared and visible modalities in face recognition.

Cutler [16] was one of the first researchers who used the eigenface [15] method in the infrared face recognition. Three views points were used in his study (frontal, 45 degree and profile) and for each view the subject had two expressions (normal and smile). He concluded that the infrared face recognition compares favourably with visible face recognition. Afterwards, many researchers used the eigenface method as a part of their face recognition system.

One of the first infrared face recognition systems was introduced by Yoshitomi et al. [17] in 1997. They focused on the lighting problem in face recognition and suggested infrared face recognition as a solution. The authors developed a method for face recognition by using Thermal Image Processing Technique.

\subsection{The important publications on Infrared Face recognition (2000-2008)}

In [18], Socolinsky et al. presented an illumination invariant face recognition system in Long-Wave Infrared imagery spectrum. In [19], the authors defined an initial framework for quantitatively analyzing the illumination invariance of thermal IR imagery of faces. They concluded that the quantitative change to thermal IR imagery of faces due to illumination changes is comparable but a little greater than changes produced by facial expression changes and temporal camera noise. They also estimated the high emissivity of human facial skin quantitatively. In [20] and [21] the authors continued their previous work in [18] by evaluating the performance of the recognition system using LDA [22], LFA [23], Eigenfaces and ICA [24] algorithms. Their results showed that LWIR imagery of human faces is superior to visible imagery in recognition. It should be noted that while their data collection included many challenging situations for visible modality, it didn't contain sufficiently challenging ones for LWIR. 
In [25] the authors used thermal infrared and visible imagery for face recognition in operational scenarios in both indoor and outdoor. They examined the performance of the system for outdoor face recognition and face recognition across multiple sessions. They collected the training set at an earlier time and in a different location. While the visible imagery was affected by changes in outdoors illumination, thermal imagery was affected both indoors and outdoors by some challenging factors such as physical activity of subjects and weather conditions. Their experimental results showed that under controlled lighting conditions, visible imagery has a better performance for indoors. For all of the algorithms that they used, outdoor recognition performance was worse for both visible and thermal modalities, with a sharper degradation for visible one. Their experiments also showed that fusion of both imaging modalities improves performance, even when they used a simple combination rule. Finally, they concluded that face recognition performance with thermal infrared imagery is stable over multiple sessions.

Chen et al. [26], [27], [28] focused on a very important factor in infrared face recognition which was the time difference between training and test images in the galleries. First they didn't consider time elapse in testing the system. In this case, they claimed that there is no consistent difference between the performance of visible and IR Infrared. Afterward, the authors focused on time elapse. They concluded that visible imagery is better than IR in time-lapsed recognition. But there was an important hint in their experiments: The sets of mismatched probes of the two classifiers for the two modalities did not overlap necessarily. This hint suggested that these two modalities may have complementary information about the probe. So, the authors combined the results of the infrared and visible based faced recognition systems using some combination rules with three different strategies. Their experimental results indicated that when there was significant time elapse (greater than one week) between the gallery and probe images, the visible face recognition outperforms the infrared one. Their experimental results also showed that the combination of the two modalities outperforms both single modalities. In [29] Sokolinsky et al. also studied the effect of time elapse on face recognition. They noticed that the thermal face recognition performance is lower than the visible one. They concluded that the difference in the performances of the two modalities is significant for a time elapse of at least six weeks.

Another important property of thermal face images were investigated by Friedrich et. al [30]. In this study, they found that the IR images are less affected by changes of pose or facial expression. They proposed an eigenfaces based method for face recognition in IR images. A pre-processing algorithm for detecting facial elements was also used in their work. They found that the IR face recognition is quite superior to visible one when the probe images include highly varying pose and facial expressions.

Pan et al. [10] used hyper-spectral images for face recognition by choosing 31 bands over the near-infrared spectrum. The reason that they used near-infrared hyper-spectral images was that spectral measurements over the nearinfrared allow the sensing of subsurface tissue structure of the skin which is unique for each person and is relatively stable over time. The authors measured the spectral variability in human skin and their results showed that there are significant differences in both amplitude and spectral shape of the reflectance curves for the different subjects, while the spectral reflectance for one subject doesn't change in different trials. The authors also concluded that the local spectral properties of human tissue are nearly invariant to face orientation and expression. This allows hyperspectral discriminants to be used for recognition of faces with different poses and expressions. In this context, the authors described an algorithm to recognize faces with varying poses and expressions over time.

In [31] the authors focused on this hint that both spatial and spectral features in hyperspectral face images are good discriminants for face recognition. It was demonstrated in [31] that the spatial features from different bands are correlated. The authors used the Principle Component Transformation (PCT) to remove this spectral redundancy between different bands. A new multiband algorithm based on spectral eigenfaces was proposed to improve face recognition performance in hyperspectral images. The new algorithm added inter-band constraints to the spatial features. Afterwards, a new multiband image, called spectral-face was proposed to preserve both spectral and spatial properties. In [32] Pan et al. used the hyperspectral imaging for face recognition with changes in head pose and facial expression when the illumination is unknown. The authors modeled the illumination variation in the radiance images by using a lowdimensional linear model for each tissue type and for each subject.

In [50] a physics-based fusion of multispectral images in the visible spectrum was proposed in order to improve face recognition under constant or varying illumination conditions. Physics properties of the imaging system, including illumination, spectral response of the camera, and spectral reflectance of skin were used to fuse the spectral images. The results showed that the multi-spectral fusion based system outperforms the system using gray-level images.

The problem with using multi-spectral images in the NIR band is that there can be big differences in the invisible spectrum in real application environments [9]. For example, there is a great difference in the infrared component between indoor and outdoor. A new approach to cope the illumination dependency problem in face recognition was proposed in [9]. They key idea behind this approach was simple. The difference was taken between two face images captured when 
the LED light is on and off. The difference image was the image of a face under just the LED illumination, and was independent of the ambient illumination.

Prokoski et al. anticipated that it is possible to extract the vascular network from thermal facial images as a feature space for face recognition [33]. Buddharaju et al. [6], [7] used this hint and presented a novel approach for face recognition based on the physiological information extracted from the thermal face images. First, they segmented the human face from the background using a Bayesian method. Then, they used image morphology to extract the blood vessels available on the segmented facial tissue. The extracted vascular network was unique for each person. The branching points of the skeletonized vascular network were referred as Thermal Minutia Points (TMPs). These TMPs were similar to the minutia points produced in the fingerprint recognition. In order to classify a test image, first the local and global structures of TMPs were extracted from the skeletonized vascular network. Afterwards, they were matched with the TMPs of the database images. This work had a drawback: The matching method could not overcome the nonlinearities in the deformation of the vascular network, due to variations in facial pose and expressions and the experimental results showed high false acceptance rates due to weaknesses of the proposed method in the feature extracting and matching algorithms.

As we know the blood vessels transporting warm blood are reflected in the thermal patterns. So, a model based on the blood perfusion can be used for face recognition. Wu et al. [8] used this hint to improve the performance of the IR face recognition system under different ambient temperatures. They converted the thermal images which are dependant to ambient temperature into the blood perfusion domain by a blood perfusion model to get more stable features against ambient temperature. In their work, the blood perfusion data were derived from the temperature distribution in the thermal patterns, and the obtained physiological data were used for face recognition. But some limitations were considered when proposing the blood perfusion model: 1-The only place of heat exchange was at the skin surface; 2-The testing subjects were in a steady state condition, and hence the deep body temperature was supposed to be constant, and no thermal regulation was considered; 3-The ambient temperature was lower than the body temperature; 4-Pathological (like fever, headache, inflammation etc) and psychological (like nervous, blush etc) conditions, were not considered; 5-The camera was well calibrated, and accurate temperature could be measured. It is obvious that all of these conditions can't simultaneously be held in real applications. After converting the thermal data into the blood perfusion domain, the features were extracted by the PCA and FLD methods and RBF neural network was used for classification. The experimental results showed that the performance of the system using blood perfusion data significantly outperforms the temperature data. In [34] the authors presented a modified blood perfusion model which was much simpler than the original one in [8]. The experimental results illustrated that the performances of the two models in [8] and [34] were comparable and both models outperformed the thermal data for the same-session data. But, the modified model had some advantage compared to the original one: 1-The modified model proposed in [34] needed less computation time than the original one; 2- When there was time elapse, the performance of the modified blood perfusion model outperformed the original one.

\section{The most Recent Works on Infrared Face Recognition (2008 to present)}

In this section we give a brief review to the state of the art on Infrared Face Recognition. Considering the previous works which were reviewed in section 2, we divide the recent works into three main categories: 1-Thermal Face Recognition; 2-Physiology Based Face Recognition; 3- Multi-Spectral Face Recognition. Our focus is on the works which have been published from 2008 to present and have not been reviewed in [1].

\section{1-Thermal Face Recognition}

A very recent study on comparison of thermal, visible and range images has been done by Mian [35]. He presented a comparison of three different modalities of the face. Unlike previous studies which used face recognition rate as a criterion, the author used the amount of variation as a comparison criterion. His conclusions were not surprising! He concluded that visible images capture more interpersonal variation in the human faces compared to thermal IR and range images. In order to reduce the intrapersonal variations in Infrared face recognition, Akhloufi and Bendada [36] proposed the use of Bayesian approaches. These approaches permit to reduce intrapersonal variation. So, they are suitable to be used in infrared face recognition. The authors used this hint and presented a new approach for face recognition in the infrared spectrum based on a probabilistic Bayesian framework [36]. Their experimental results showed that the proposed technique gives promising results when sufficient number of face images is used in an intrapersonal learning process. 
As mentioned before, thermal face recognition has attracted the most attention in the Infrared Face Recognition. One of the recent works on this field has been presented by Bhowmik et al. [37]. They used Log-polar transform to achieve rotation and scaling invariant images. Afterwards, they applied the eigenface (PCA) algorithm to these transformed images and used a multilayer perceptron for classification. In [38] the authors claimed that with the presence of some factors such as illuminations and facial expressions, the linear dimensionality reduction methods such as PCA cannot model the data efficiently. They suggested using Kernel approach to overcome these limitations. For this reason, they examined Kernel Principle Component Analysis (KPCA) and Kernel Fisher's Linear Discriminant Analysis (KFLD). The main contribution of their work was a fusion scheme performed at nonlinear transformed domain. The IR and visible feature components were extracted by the kernel methods and then were fused using GA (Genetic Algorithms) for obtaining an optimal fusion of both modalities. Their experimental results showed that using GA as the combination method and KFLD as the feature extracting method has the best performance for simultaneously handling the problems of single modality face recognition and linear dimension reduction methods.

Another method to combine the visual and thermal face images for face recognition was presented in [39]. The method consisted of a feature extracting stage and a fusion stage. The feature extraction stage was the combination of a high dimensional random projection with a feature regularization and extraction process. The fusion stage was performed using a proposed error counting algorithm [39]. Their experimental results showed that useful information can be extracted based on a high dimensional random projection with eigenvalue regularization. The results also showed that the fusion improves the performance compared to the single modalities at low polynomial model orders.

\section{2- Physiology Based Face Recognition}

In section 2 we indicated that the authors in [8] used blood perfusion data instead of thermal data for face recognition. They examined the recognition system with PCA and FLD methods as feature extracting algorithms. As mentioned before, those feature extracting methods cannot model the data efficiently with the presence of some factors such as illuminations and facial expressions. They also suffer from some other problems such as complexity of computation, large memory units and small samples. To overcome these latter problems, some researchers have proposed the face recognition methods based on sparse representation [40]. Curvelet Transformation [41] is an effective sparse representation method that has a good directional and edge demonstration abilities which makes it suitable to be used in infrared face recognition. In [42]-[44], the authors proposed a fast infrared face recognition system using Curvelet transformation. First thermal images were converted into blood perfusion data. Afterwards, the converted images were decomposed into their Curvelet subbands to extract the principal features. Finally, the nearest neighbor algorithm was used for classification. The experimental results showed that the proposed system has better performance than the PCA based systems and it requires less computations and memory units.

In [45], Buddharaju et al. modified their previous work [7] in order to solve its indicated drawbacks. A new vessel segmentation post-processing algorithm that removes fake vascular contours was proposed in [45]. A new vascular network matching algorithm that is robust to non-linear deformations due to facial pose and expression variations was also proposed. The new matching method used the dual bootstrap iterative closest point (ICP) matching algorithm [46] in order to register test and database vascular networks. The authors compared their experiments with their previous work to evaluate the performance of the new method. The results showed that the dual bootstrap ICP is superior to the TMP matching algorithm. The results also confirmed that the new algorithm show good performance when there are facial pose variations between gallery and test images.

In [47], the authors tried to improve the robustness of the thermal face patterns as a biometrics by refining the Buddahraju's approach by proposing the bifurcation points of the thermal pattern and the geographical gravity center of the thermal face region as some extra features in addition to the minutiae points. A bifurcation point is a place in the blood vessel in which the branching appears. Bifurcation points can be extracted from the blood vessel network in a similar way as fingerprint. The authors suggested using the Modified Hausdorff Distance (MHD) [48] to measure the similarity between feature sets. Their experimental results showed that using geographical gravity center as an extra feature improves the accuracy of the system significantly. They concluded that the thermal face patterns have a reasonable discriminating power as a biometric, but their accuracy is lower than other main biometrics.

Akhloufi and Bendada [49] proposed an approach for face recognition in the infrared spectrum by using thermal face prints. In the proposed approach, physiological features were extracted from the thermal images in order to build a thermal face print. The obtained physiological features are related to the distribution of blood vessels under the face skin. The authors claimed that the Face print is unique for each person and can be used in face recognition. An Euclidian distance transform was applied to the face prints to get an invariant representation for face recognition. The 
Euclidian distance between the distance transformed images was used in order to classify the transformed images in the face recognition system. The authors claimed that promising results were achieved.

\section{3- Multi-Spectral Face Recognition}

Multispectral imaging sensors have the capability of measuring the spectral information within a wide range of wavelengths. By using the multispectral imaging, one is capable to get an image with emphasize and/or suppress the contribution of the images from distinct narrow spectral bands. As it was indicated before, Pan et al. [10] used nearinfrared hyper-spectral images for face recognition with different pose and expressions. But they did not consider the illumination changes in their work. In order to overcome the illumination changes problem, Chang et al. [11], [51] proposed using narrowband spectral images instead of broadband images. An automated band selection algorithm was developed to choose the optimal band images under given illumination conditions. The authors believed that certain spectral bands contain more information than the others for multispectral image fusion. In this context, they proposed an illumination-specific spectral range selection algorithm that defines a minimum set of narrow spectral bands to improve face recognition performance in the visible domain. In [52] the authors discussed about a framework for multispectral and thermal imaging system in the visual and infrared spectrum. They also studied on multi-spectral and thermal image fusion. They concluded that a subset of spectral bands can be selected for fusion without degradation in face recognition performance by using the weight values determined based on the filter transmittance, spectral response of imaging sensor, illumination distribution, and skin reflectance.

\section{Conclusions}

In this paper, a review on the state of the art in Infrared Face Recognition has been done. The main reason that the researchers have been interested in face recognition is that the identity recognition systems based on the other biometrics need the cooperation of the subject with the system, but this is not the case for the face biometric. One of the main problems in the visible face recognition is the illumination dependency problem. In order to solve this problem, the use of Infrared face recognition has been suggested. Among the infrared sub-bands, Thermal infrared and Near Infrared have received more attention than Short Wavelength and Medium Wave length infrared.

Thermal infrared has some attractive properties which makes it interesting for face recognition. For example, it is not subject to the illumination changes of the environment. On the other hand it is somewhat robust to facial expression and the pose of the face. It also reveals anatomical information of face that is useful in detecting disguised faces. But, thermal infrared has some drawbacks. It is subject to environmental temperature, emotional, physical and health conditions. Another problem of the thermal infrared is the opaqueness to eyeglasses which make a large portion of the face wearing eyeglasses to be occluded. This tends some information around the eyes not to be accessible in the thermal face images. It has been also shown that thermal face recognition doesn't show satisfying results when there is a large time elapse between testing and training data. In order to solve the opaqueness problem, most of the researchers have suggested the fusion of information from visible with thermal data. In order to solve the environmental temperature dependency problem of the thermal images, the use of physiological information of the face has been suggested.

As another way to overcome the illumination dependency problem in visible face recognition, some researchers have proposed the use of active near infrared, because of the mentioned limitations of the thermal imagery. An important property of NIR spectrum is its invisibility to human eyes which makes it unobtrusive. Recently, the use of Hyperspectral imagery in both Near Infrared and Visible bands has been suggested to overcome the illumination dependency problem of traditional face recognition systems. Multispectral imaging sensors have the capability of measuring the spectral information within a wide range of wavelengths. By using the multispectral imaging, one is capable to get an image with emphasize and/or suppress the contribution of the images from distinct narrow spectral bands.

LWIR and SWIR have not been investigated very well in the Face Recognition. With the decrease in the cost of the infrared cameras, and the great potential of the infrared face recognition in solving the problems of the visible face recognition, a growing research interest in each of the four infrared sub bands is expected in future.

\section{REFERENCES}

[1] Moulay Akhloufi, Abdel Hakim Bendada and Jean-Christophe Batsale, "State of the art in infrared face recognition", Quantitative Infrared Thermography Journal (QIRT), vol. 5, no 1, pp. 3-26, 2008. 
[2] Zhao W., Chellappa R., Phillips P., Rosenfeld A., "Face recognition: A literature survey", ACM Computing Surveys, vol. 35, p. 399-458, 2003.

[3] S.Z. Li et al., "AuthenMetric F1: A Highly Accurate and Fast Face Recognition System," Proc. Int'l Conf. Computer Vision, Oct. 2005.

[4] Pavlidis, I. and Symosek, P. 2000. The imaging issue in an automatic face/disguise detection system. In Proc. IEEE Workshop on Computer Vision Beyond the Visible Spectrum: Methods and Applications, pp. 15-24.

[5] Kong S., Heo J., Abidi B., Paik J., Abidi M., "Recent advances in visual and infrared face recognition: a review", Computer Vision \& Image Understanding, vol. 97, p. 103-135, 2005.

[6] P. Buddharaju, I. Pavlidis, and P. Tsiamyrtzis, "Phsiology-based face recognition using the vascular network extracted from thermal facial images: A novel approach ", Proceedings of the IEEE International Conference on Advanced Video and Signal based Surveillance, Como, Italy, September 15-16, 2005.

[7] P. Buddharaju, I.T. Pavlidis, P. Tsiamyrtzis and M. Bazakos, "Physiology-Based face Recognition in the Thermal Infrared Spectrum", IEEE Transactions on Pattern Analysis and Machine Intelligence, vol.29, No. 4, pp. 613-626, April 2007.

[8] WU, S., SONG, W., JIANG, L.J., XIE, S., PAN, F., YAU, W.Y., AND RANGANATH, S., "Infrared face recognition by using blood perfusion data," In Proceedings of the Intl Conf Audio Video Based Biometric Person Authentication (New York, USA., July 20-22, 2005.) Pp. 320-328.

[9] X. Zou, J. Kittler, and K. Messer, "Face Recognition Using Active Near-IR Illumination," Proc. British Machine Vision Conf., Sept. 2005.

[10] Z. Pan and G. Healey, "Face recognition in hyperspectral images," IEEE Trans. Pattern Anal. Mach. Intell. 25, pp. $1552-1560$ (2003).

[11] H. Chang, Y. Yao, A. Koschan, B. Abidi, and M. Abidi, "Improving Face Recognition via Narrowband Spectral Range Selection Using Jeffrey Divergence" IEEE TRANSACTIONS ON INFORMATION FORENSICS AND SECURITY, VOL. 4, NO. 1, MARCH 2009.

[12] F. J. Prokoski, R. B. Riedel, and J. S. Coffin, "Identification of individuals by means of facial thermography," in Proceedings of The IEEE 1992 International Carnahan Conference on Security Technology: Crime Countermeasures, Atlanta, GA, USA 14-16 Oct., pp. 120-125, IEEE, 1992.

[13] F.J. Prokoski, Method for identifying individuals from analysis of elemental shapes derived from biosensor data, US Patent 5,163,094, November 1992.

[14] Wilder J., Phillips P., Jiang C., Wiener S., "Comparison of visible and infra-red imagery for face recognition", Proceedings of the IEEE International Conference on Automatic Face and Gesture Recognition (AFGR '96), p. 182187, Killington, Vermont, October, 1996.

[15] Turk, M., and Pentland, A., "Eigenfaces for Recognition," Journal of Cognitive Neuroscience, Vol. 3, No. 1, pp. 7186, 1991.

[16] Cutler R., "Face recognition using infrared images and eigenfaces," http://citeseer.ist.psu.edu/cutler96face.html, April, 1996, visited July 2007.

[17] Y. Yoshitomi, T. Miyaura, S. Tomita, S. Kimura, Face identification using thermal image processing, Proc. IEEE Int. Workshop Robot Hum. Commun. (1997) 374-379.

[18] D.A. Socolinsky, L.B. Wolff, J.D. Neuheisel, and C.K. Eveland, "Illumination Invariant Face Recognition Using Thermal Infrared Imagery," Proc. IEEE CS Conf. Computer Vision and Pattern Recognition, vol. 1, pp. 527-534, Dec. 2001.

[19] L. B. Wolff, D. A. Socolinsky, and C. K. Eveland, "Quantitative measurement of illumination invariance for face recognition using thermal infrared imagery," Proc. CVPR Workshop on Computer Vision Beyond the Visible Spectrum, 2001.

[20] Socolinsky, D. and Selinger A., "Comparative study of face recognition performance with visible and thermal infrared imagery," in International Conference on Pattern Recognition, pp. 217-222, 2002.

[21] A. Selinger, D. Socolinsky, Appearance-based facial recognition using visible and thermal imagery: a comparative study, Equinox Corporation no. 02-01, Technical Report, 2002.

[22] P. Belhumeur, J. Hespanha, and D. Kriegman, "Eigenfaces vs. Fisherfaces: Recognition Using Class Specific Linear Projection," IEEE Transactions PAMI, vol. 19, no. 7, pp. 711-720, July 1997.

[23] P. Penev and J. Attick, "Local Feature Analysis: A general statistical theory for object representation," Network: Computation in Neural Systems, vol. 7, no. 3, pp. 477-500, 1996.

[24] Liu C., Wechsler H., "Comparative assessment of independent component analysis (ICA) for face recognition", Proceedings of the International Conference on Audio and Video- Based Biometric Person Authentication (AVBPA'99), p. 211-216, Washington D.C., March, 1999.

[25] D. A. Socolinsky and A. Selinger, "Thermal face recognition in an operational scenario," in Proceedings of CVPR 2004, Washington, D.C., 2004.

[26] Chen, X. and Flynn, P. and Bowyer, K., "PCA-based face recognition in infrared imagery: Baseline and comparative studies," in IEEE International Workshop on Analysis and Modeling of Faces and Gestures, (Nice, France), 2003. 
[27] Chen, P. J. Flynn and K. W. Bowyer, "IR and visible light face recognition", Computer Vision and Image Understanding, vol. 99, pp. 332-358, 2005.

[28] X. Chen, P. Flynn, K. Bowyer, Visible-light and infrared face recognition, in: Proc. Workshop on Multimodal User Authentication, 2003, pp. 48-55.

[29] Socolinsky, D. A. \& Selinger. A. (2004B). Thermal face recognition over time, Proceedings of Int. Conf. Pattern Recognition, pp. 187-190, Cambridge, UK, 2004.

[30] G. Friedrich, Y. Yeshurun, "Seeing people in the dark: face recognition in infrared images," in: Second BMCV, 2003.

[31] Z. Pan, G. Healey, M. Prasad, and B. Tromberg, "Multiband and spectral eigenfaces for face recognition in hyperspectral images," in Proc. SPIE, 2005, vol. 5779, pp. 144-151.

[32] Z. Pan, G. Healey, M. Prasad, and B. Tromberg, "Hyperspectral face recognition under variable outdoor illumination," Proceedings of SPIE, Vol. 5425, pp. 520-529, 2004.

[33] F.J. Prokoski and R. Riedel. BIOMETRICS: Personal Identification in Networked Society, chapter 9 Infrared Identification of Faces and Body Parts. Kluwer Academic Publishers, 1998.

[34] Shiqian Wu, Zhenghui Gu, Kia Ai Chia, Sim Heng Ong, "Infrared Facial Recognition Using Modified Blood Perfusion", 6th International Conference on Information, Communications \& Signal Processing, 2007.

[35] Ajmal Mian," Comparison of Visible, Thermal Infra-Red and Range Images for Face Recognition" Lecture Notes In Computer Science; Vol. 5414 archive Proceedings of the 3rd Pacific Rim Symposium on Advances in Image and Video Technology.

[36] Moulay A. Akhloufi, and Abdelhakim Bendada, "Probabilistic Bayesian framework for infrared face recognition," World Academy of Science, Engineering and Technology 602009.

[37] Mrinal Kanti Bhowmik, Debotosh Bhattacharjee, Mita Nasipuri, Dipak Kumar Basu Mahantapas Kundu, "CLASSIFICATION OF POLAR-THERMAL EIGENFACES USING MULTILAYER PERCEPTRON FOR HUMAN FACE RECOGNITION" 2008 IEEE Region 10 Colloquium and the Third ICIIS, Kharagpur, INDIA December 8-10.

[38] Shahbe M. Desa and Subhas Hati, "IR and Visible Face Recognition using Fusion of Kernel Based Features,"19th International Conference on Pattern Recognition, 2008. ICPR 2008.

[39] Byung-Gue Choi, Youngsung Kim and Kar-Ann Toh, "A Method to Combine Visual and Infrared Face Image Verification Systems" 2008 10th Intl. Conf. on Control, Automation, Robotics and Vision Hanoi, Vietnam, 17-20 December 2008.

[40] Allen Y. Yang, John Wright, Yi Ma, "Feature Selection in Face Recognition: A Sparse Representation Perspective", MANUSCRIPT SUBMITTED TO IEEE TRANS. PAMI, AUGUST 2007.

[41] T. Mandal, A. Majumdar, Q.M. J. Wu Face Recognition by Curvelet Based Feature Extraction, Proc of ICIAR, Vol. 4633, 2007, p. 806-817.

[42] Zhihua Xie, Guodon Liu, Shiqian Wu, Yu Lu "A Fast Infrared Face Recognition System Using Curvelet Transformation" Second International Symposium on Electronic Commerce and Security, 2009. ISECS '09.

[43] Xie, Shiqian Wu, Guodong Liu, Zhijun Fang, "Infrared Face Recognition Based on Radiant Energy and Curvelet Transformation" 2009 Fifth International Conference on Information Assurance and Security.

[44] Xie, Shiqian Wu, Guodong Liu, Zhijun Fang, "INFRARED FACE RECOGNITION METHOD BASED ON BLOOD PERFUSION IMAGE AND CURVELET TRANSFORMATION" Proceedings of the 2009 International Conference on Wavelet Analysis and Pattern Recognition, Baoding, 12-15 July 2009

[45] Pradeep Buddharaju loannis Pavlidis, "Physiological face recognition is coming of age", IEEE Conference on Computer Vision and Pattern Recognition, 2009. CVPR 2009.

[46] C. V. Stewart, C. Tsai, and B. Roysam. The dual-bootstrap iterative closest point algorithm with application to retinal image registration. IEEE Transactions on Medical Imaging, 22(11):1379-1394, 2003.

[47] Siu-Yeung Cho, Lingyu Wang and Wen Jin Ong "Thermal Imprint Feature Analysis for Face Recognition" IEEE International Symposium on Industrial Electronics (ISIE 2009) Seoul Olympic Parktel , Seoul, Korea July 5-8, 2009

[48] M. Dubuisson \& A. Jain, "A modified hausdorff distance for object matching", In Proceedings of 12th international conference on pattern recognition. pp. 566-568. Jerusalem, Iserael.

[49] M. Akhloufi, A. Bendada, "Infrared face recognition using distance transforms", ICIVC 2008: International Conference on Image and Vision Computing, Paris, France, July 04-06, 2008, Proceedings of World Academy of Science, Engineering \& Technology, Vol. 30, ISSN 1307-6884, pp. 160-163.

[50] H. Chang, A. Koschan, B. Abidi, and M. Abidi, "Physics-based fusion of multispectral data for improved face recognition," IEEEProc. ICPR2006, vol. III, pp. 1083-1086, 2006.

[51] H. Chang, Y. Yao, A. Koschan, B. Abidi, and M. Abidi, "Spectral Range Selection for Face Recognition under Various Illuminations," Proc. Int'l Conference on Image Processing, 2008.

[52] H. Chang, A. Koschan, M. Abidi, S. G. Kong, C. Won, "Multispectral Visible and Infrared Imaging for Face Recognition", IEEE Computer Society Conference on Computer Vision and Pattern Recognition Workshops, 2008. 